\title{
Long Noncoding RNA FOXP4-AS1 Predicts Unfavourable Prognosis and Regulates Proliferation and Invasion in Hepatocellular Carcinoma
}

\author{
Jingchen Liang $\mathbb{D}$, Duo Wang, Guanhua Qiu, Xiaoqi Zhu, Junjie Liu $\mathbb{D}$, Hang Li $\mathbb{D}$, \\ and Pingping Guo
}

\author{
Department of Ultrasound, Affiliated Tumor Hospital of Guangxi Medical University, Nanning, \\ Guangxi Zhuang Autonomous Region 530021, China
}

Correspondence should be addressed to Junjie Liu; liujunjie197806@163.com, Hang Li; lihang58@126.com, and Pingping Guo; guopingpinggmu@163.com

Jingchen Liang, Duo Wang, and Guanhua Qiu contributed equally to this work.

Received 17 September 2020; Revised 4 January 2021; Accepted 17 January 2021; Published 1 February 2021

Academic Editor: Fu-Ming Tsai

Copyright (C) 2021 Jingchen Liang et al. This is an open access article distributed under the Creative Commons Attribution License, which permits unrestricted use, distribution, and reproduction in any medium, provided the original work is properly cited.

\begin{abstract}
Background. Hepatocellular carcinoma (HCC) is the most common type of primary liver cancer that has a high level of morbidity and mortality. Long noncoding RNA (lncRNA) is a novel regulatory factor of tumour proliferation, apoptosis, and metastasis. Our previous studies indicated that lncRNA FOXP4-AS1 is a functional oncogene in HCC; thus, this study is aimed at further evaluating the clinical and biological function of FOXP4-AS1 in HCC. Material and Methods. First, we detected the expression of FOXP4-AS1 in HCC tissues and paracarcinoma normal tissues by qRT-PCR. Second, the prognostic effects of FOXP4-AS1 in patients with HCC were analysed in a training group and a verification group. Subsequently, to investigate the biological effects of FOXP4-AS1 on HCC cells, downexpression tests were further conducted. Results. The expression of FOXP4-AS1 was higher in HCC tissues than adjacent nontumourous tissues, whereas the low expression of FOXP4-AS1 was correlated with optimistic treatment outcomes, which suggested that FOXP4-AS1 may be an independent prognostic biomarker for HCC. Moreover, the downregulation of FOXP4-AS1 significantly reduced the cell proliferation and clonal abilities and inhibited the invasion, migration, and angiogenesis of hepatoma cells $(P<0.05)$. Conclusion. These results revealed the clinical significance and biological function of FOXP4-AS1 in HCC development, which may provide a new direction for finding therapeutic targets and potential prognostic biomarkers of HCC.
\end{abstract}

\section{Introduction}

Hepatocellular carcinoma (HCC) is one of the most common malignant tumours worldwide. Approximately 700,000 new cases are diagnosed every year [1]. Although great progress has been made in the treatment for HCC in recent years, including surgical resection, liver transplantation, radiofrequency ablation, interventional therapy, and drug targeted therapy, the overall prognosis of patients with HCC remains poor owing to the high rates of recurrence and metastasis [2]. Therefore, it is essential to study the pathogenesis of liver cancer and finding tumour markers with high sensitivity and specificity to provide a more reliable basis for the early diagnosis, effective treatment, and prognosis of liver cancer.

Recently, long noncoding RNAs (lncRNAs) have gradually entered the field of vision of researchers. IncRNAs are a type of noncoding RNA with $>200$ nucleotides; lncRNAs can affect many biological processes such as epigenetic modification, transcription regulation, protein translation, and degradation [3, 4]. More and more new lncRNAs have been found and identified as tumour suppressor genes in human cancer, which provides the possibility that lncRNAs may function as new tumour biomarkers and therapeutic targets [5, 6]. Currently, lncRNA forkhead box P4 antisense RNA 
1 (FOXP4-AS1), which is a lncRNA related to tumours, is believed to participate in the occurrence of tumours and promote tumour proliferation, invasion, and migration; thus, its upregulation is usually related to tumour grade and poor prognosis $[7,8]$. Nevertheless, the capability of FOXP4-AS1 in the development of HCC remains unclear.

This study is aimed at detecting the FOXP4-AS1 expression level in HCC tissues and at investigating its effect on prognosis. Furthermore, in vitro experiments are aimed at examining the impact of FOXP4-AS1 on the biological function of HCC cells.

\section{Materials and Methods}

2.1. Clinical Sample Collection. A total of 121 pairs of surgical specimens, containing tumour tissues and paracarcinoma normal tissues, from patients with liver cancer, were collected from the Affiliated Tumour Hospital of Guangxi Medical University. All patients in the group had no loss of followup and complete clinicopathological data. Among them, the training group recruited 87 cases from January 2014 to December 2014, and the validation group enrolled 34 patients from January 2015 to December 2015. All patients underwent radical hepatectomy and were diagnosed as having HCC by pathology and did not receive radiotherapy or chemotherapy before surgery. The adjacent normal liver tissues were collected $>2 \mathrm{~cm}$ away from the tumour boundary, and the tumour-free liver tissues were confirmed by pathology. The collected tissues were immediately stored in a refrigerator at $-80^{\circ} \mathrm{C}$. This study was ratified and supervised by the ethics committee of our hospital.

2.2. Cell Lines and Transfection. The human hepatoma cell lines Hep3B and Huh7 were preserved in our laboratory. The cells were cultured in Dulbecco's modified Eagle's medium supplemented with $10 \%$ foetal bovine serum (Gibco, USA) and grown in a wet incubator at $37^{\circ} \mathrm{C}$ and $5 \% \mathrm{CO}_{2}$. With the assistance of Shanghai GeneChem Co., Ltd., the siRNA target sequence design was completed $(3 \mathrm{E}+8 \mathrm{TU} / \mathrm{ml})$. Cell transfection was performed using a transfection reagent (Thermo Scientific, USA) according to the manufacturer's instructions. After 48 hours of transfection, the cells were collected for further study.

The experimental transfection of the siRNA sequence is as follows: FOXP4-AS1-siRNA:5' - TGGCAACCTAGTAA CCATTAA- ${ }^{\prime}$; scramble-siRNA: $5^{\prime}{ }^{\prime}$ TTCTCCGAACG TGTCACGT-3'.

2.3. $q R T-P C R$. RNA from tissue samples and cells was extracted using the TRIzol reagent (Thermo Scientific, USA). Subsequently, the qualified RNA was retrotranscribed into cDNA using the PrimaScript ${ }^{\mathrm{TM}}$ RT Reagent Kit with gDNA Eraser (Takara Biotechnology, Dalian, China). The RNA expression was detected by RT-PCR using the FastStart Universal SYBR Green Master (Rox) kit (Roche, Germany). The correlation expression level was calculated by the Livak $(2-\Delta \mathrm{CT})$ method with $\beta$-actin as a reference. The primer sequences of FOXP4-AS1 were $5^{\prime}$-TCGGGTGGAAGTCG TTGC- $3^{\prime}$ and $5^{\prime}$ - CCTCCGCTTGTCTCCCTTT- $3^{\prime}$; the primer sequences of $\beta$-actin were $5^{\prime}$-TGCGTGACATTAAG GAGAAG- $3^{\prime}$ and $5^{\prime}$-GTCAGGCAGCTCGTAGCTCT-3'.

2.4. CCK-8 Assay. Cell Counting Kit-8 (CCK-8, Tongren, China) was utilized to evaluate cell proliferation. The cells were inoculated in a 96-well plate at a density of $2 \times 10^{3}$ cells/well and cultured for the indicated times $(24,48,72$, 96, or 120 hours). Afterwards, $10 \mu \mathrm{l}$ of CCK- 8 solution was added into each pore and incubated at $37^{\circ} \mathrm{C}$ for 100 minutes. The absorbance at $450 \mathrm{~nm}$ was measured using a Thermo enzyme reader (Thermo Fisher Scientific, USA).

2.5. Colony Formation Assay. A total of 500 hepatoma cells per well were inoculated into 6-well plates. Colony formation after routine culture was conducted for 2 weeks. Cells were washed with phosphate-buffered saline, fixed with methanol, and then stained with $0.1 \%$ crystal violet (Sinopharm Chemical Reagent Co., Ltd., China). Visible colonies were counted and imaged by the microscope.

2.6. Wound Healing Assay. Hepatoma cells were inoculated into 6-well plates and then incubated overnight. The wound was first scratched with the tip of a $200-\mu$ l straw and then cultured in serum-free medium mixed with cytarabine $(5 \mu \mathrm{mol} / \mathrm{l})$. Photos were taken at 0,24 , and 48 hours ( $\geq 3$ visual fields/hour), and the migration area of each group of photos was calculated with the ImageJ software. Later, the migration area was calculated according to the following formula: migration area $(\mathrm{nh})=$ blank area $(0 \mathrm{~h})-$ blank area $(\mathrm{nh})$.

2.7. Transwell Assay. The cell density was adjusted to $2 \times$ $10^{5}$ cells $/ \mathrm{ml}$ with serum-free medium, then added $100 \mu \mathrm{l}$ to the upper chamber of the transwell. Meanwhile, $500 \mu \mathrm{l}$ of complete medium was placed into the lower chamber and then cultured for 48 hours. The cells were routinely fixed, stained, and then imaged using a fluorescence inverted routine microscope (Olympus, Japan) in 5 random 200x microscopic fields. The crystal violet at the bottom of the chamber was eluted with $300 \mu \mathrm{l}$ of $33 \%$ acetic acid. Notably, $100 \mu \mathrm{l}$ was added into each well of the 96-well plates, and the OD value at $590 \mathrm{~nm}$ was read using an enzyme labelling instrument. Different from the migration experiment, the invasion experiment was coated with a layer of Matrigel matrix (50 $\mu \mathrm{l}, 1-1.5 \mathrm{mg} / \mathrm{ml})$ (Corning, USA) in the transwell chamber. The rest of the operation is the same as the migration test.

2.8. Angiogenesis Assay. Different groups of cells were seeded on a 6-well plate and cultured in serum-free medium for 24 hours after adhering to the wall, then collected the supernatant culture medium. Human umbilical vein endothelial cells (HUVECs) were digested and centrifuged and then suspended with a preestablished supernatant medium and inoculated into a 24-well plate coated with $70 \mu \mathrm{l}$ Matrigel $^{\mathrm{TM}}$ matrix $(8-12 \mathrm{mg} / \mathrm{ml})(\mathrm{BD}, \mathrm{USA})$ at $1.5 \times 10^{4} /$ well and cultured in a carbon dioxide incubator at $37^{\circ} \mathrm{C}$ for 24 hours. Then, calcein was added to the culture and photographed.

2.9. Statistical Analysis. The SPSS 24.0 software (SPSS, Chicago, IL, USA) was used for data analysis, whereas the 


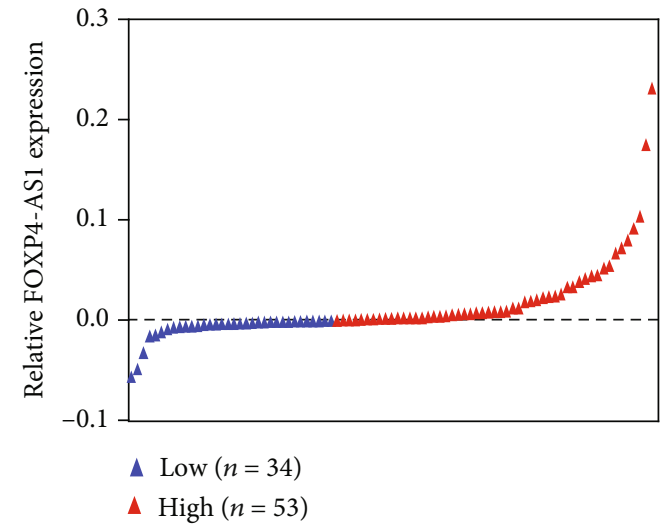

(a)

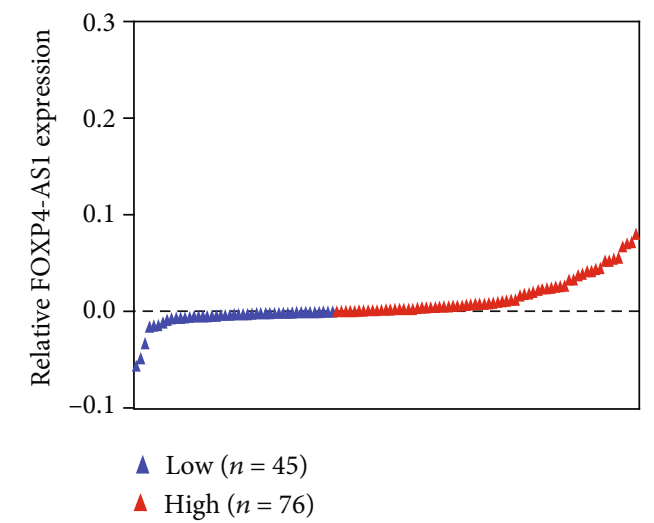

(c)

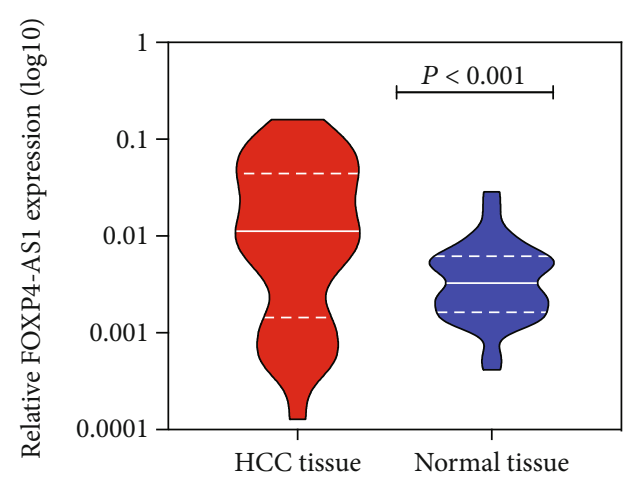

(e)

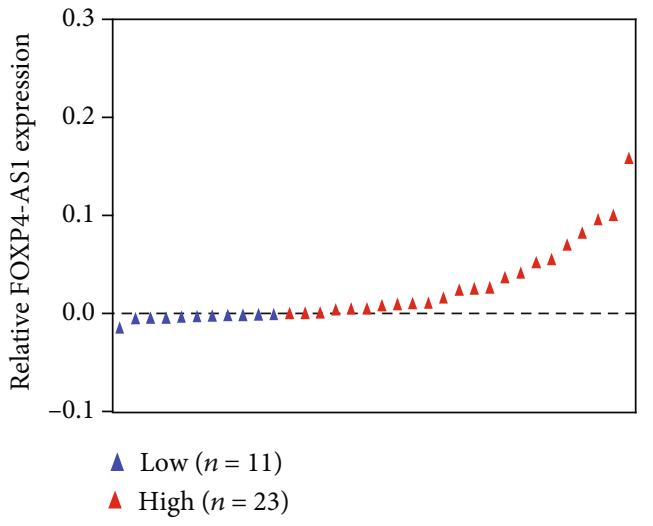

(b)

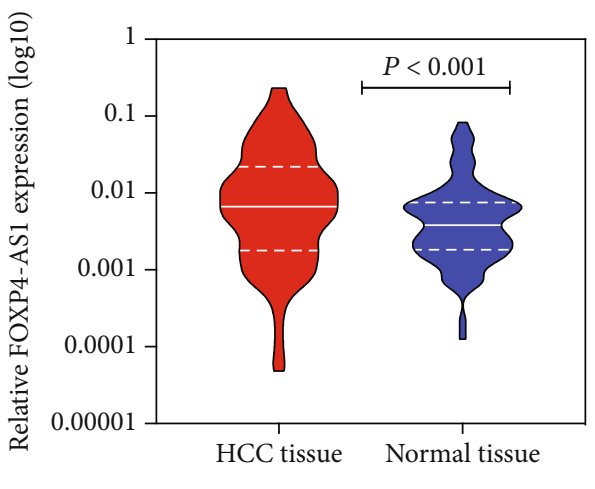

(d)

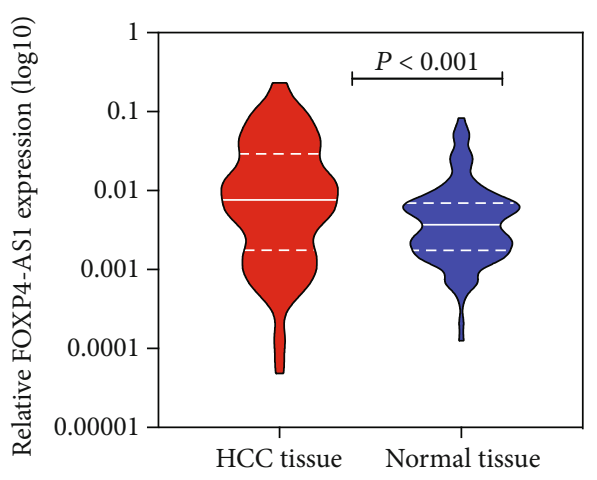

(f)

Figure 1: Continued. 


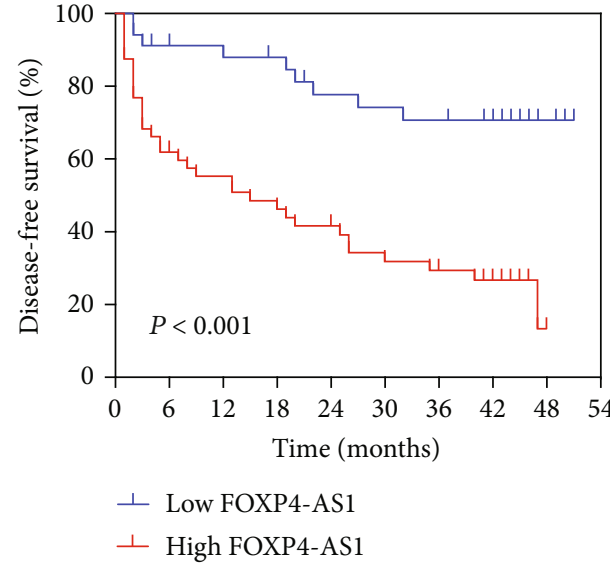

(g)

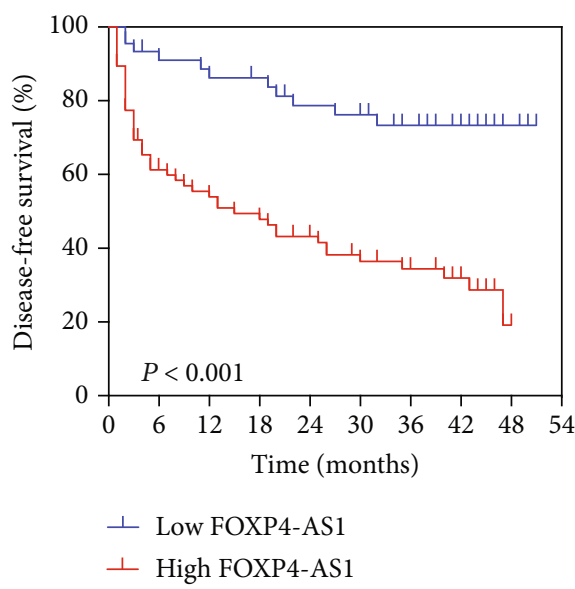

(i)

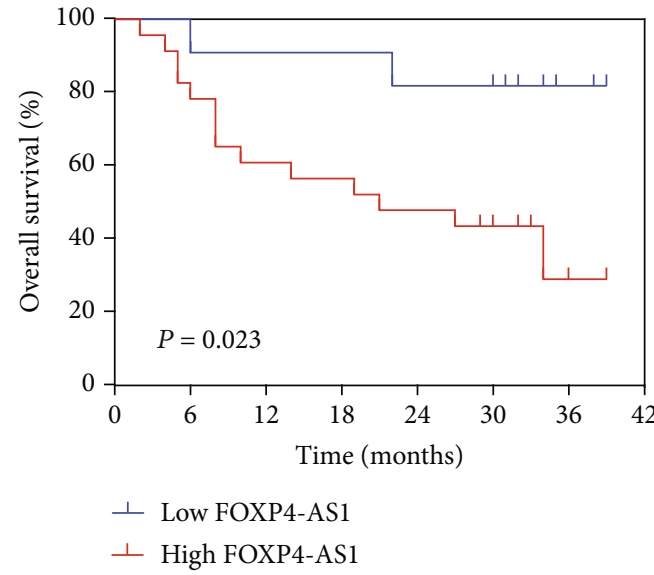

(k)

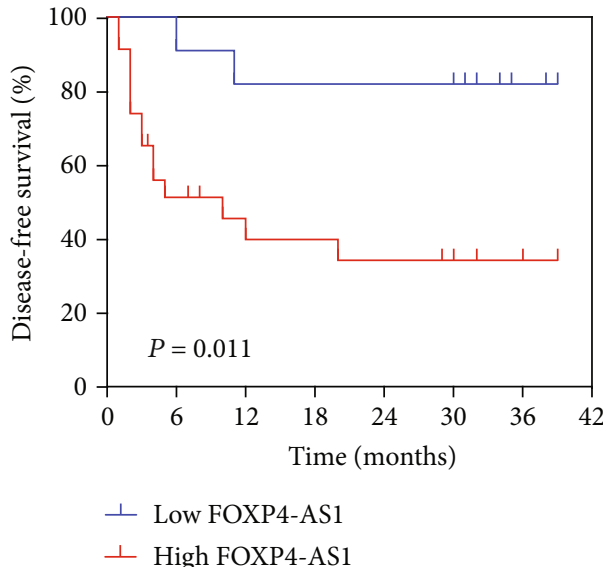

(h)

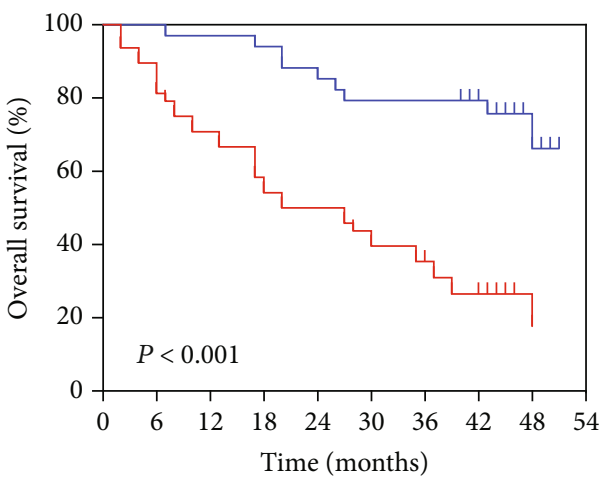

$\perp$ Low FOXP4-AS1

$\perp$ High FOXP4-AS1

(j)

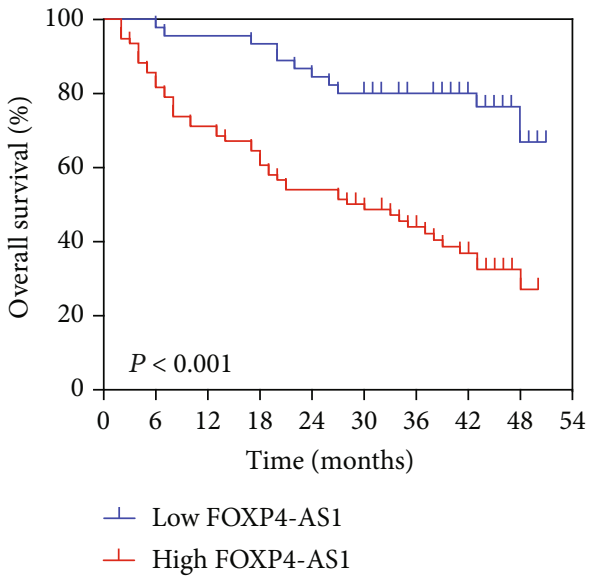

(1)

FIgURE 1: Expression of FOXP4-AS1 in HCC and its correlation with clinical outcome. Patients were divided into groups based on the difference of FOXP4-AS1 expression between HCC and adjacent normal tissues: (a) training set, (b) validation set, and (c) combined set. Compared with adjacent nontumor tissues, the FOXP4-AS1 expression was downregulated in the training set (d), validation set (e), and combination set (f) using the qRT-PCR assay. The DFS and OS of HCC patients with high or low FOXP4-AS1 expression in the training set $(n=87 ;(\mathrm{g}),(\mathrm{j})$, validation set $(n=34 ;(\mathrm{h}),(\mathrm{k}))$ and combined set $(n=121 ;(\mathrm{i}),(\mathrm{l}))$ were compared by the Kaplan-Meier survival analysis.

graphics were created by the GraphPad Prism 8 software (GraphPad Software Inc., La Jolla, CA, USA). T-test, $\chi^{2}$ tests, and multivariate Cox regression analysis were used to assess the significant differences between the data groups. All data were expressed as the mean \pm standard of the mean. $P<0.05$ was considered statistically significant. 
TABLE 1: Clinicopathological features by FOXP4-AS1 expression levels.

\begin{tabular}{|c|c|c|c|c|c|c|c|c|c|c|c|c|}
\hline \multirow[b]{2}{*}{$\begin{array}{l}\text { Clinicopathologic } \\
\text { parameters }\end{array}$} & \multicolumn{4}{|c|}{ Training set } & \multicolumn{4}{|c|}{ Validation set } & \multicolumn{4}{|c|}{ Combined set } \\
\hline & $\begin{array}{c}\text { Total } \\
(n=87)\end{array}$ & $\begin{array}{c}\text { Low } \\
(n=34)\end{array}$ & $\begin{array}{c}\text { High } \\
(n=53)\end{array}$ & $P$ value & $\begin{array}{c}\text { Total } \\
(n=34)\end{array}$ & $\begin{array}{c}\text { Low } \\
(n=11)\end{array}$ & $\begin{array}{c}\text { High } \\
(n=23)\end{array}$ & $P$ value & $\begin{array}{c}\text { Total } \\
(n=121)\end{array}$ & $\begin{array}{c}\text { Low } \\
(n=45)\end{array}$ & $\begin{array}{c}\text { High } \\
(n=76)\end{array}$ & $P$ value \\
\hline Gender & & & & 0.505 & & & & 0.239 & & & & 0.978 \\
\hline Female & 13 & 4 & 9 & & 3 & 2 & 1 & & 16 & 6 & 10 & \\
\hline Male & 74 & 30 & 44 & & 31 & 9 & 22 & & 105 & 39 & 66 & \\
\hline Age (years) & & & & 0.001 & & & & 0.580 & & & & 0.001 \\
\hline$<60$ & 69 & 21 & 48 & & 30 & 9 & 21 & & 99 & 30 & 69 & \\
\hline$\geq 60$ & 18 & 13 & 5 & & 4 & 2 & 2 & & 22 & 15 & 7 & \\
\hline Family history & & & & 0.142 & & & & 0.580 & & & & 0.532 \\
\hline No & 79 & 33 & 46 & & 30 & 9 & 21 & & 109 & 42 & 67 & \\
\hline Yes & 8 & 1 & 7 & & 4 & 2 & 2 & & 12 & 3 & 9 & \\
\hline AFP & & & & 0.002 & & & & 0.138 & & & & $<0.001$ \\
\hline$<400 \mathrm{ng} / \mathrm{ml}$ & 43 & 24 & 19 & & 12 & 6 & 6 & & 55 & 30 & 25 & \\
\hline$\geq 400 \mathrm{ng} / \mathrm{ml}$ & 44 & 10 & 34 & & 22 & 5 & 17 & & 66 & 15 & 51 & \\
\hline HBVDNA & & & & 0.478 & & & & 1.000 & & & & 0.422 \\
\hline Negative & 22 & 10 & 12 & & 8 & 3 & 5 & & 30 & 13 & 17 & \\
\hline Positive & 65 & 24 & 41 & & 26 & 8 & 18 & & 91 & 32 & 59 & \\
\hline Metastasis & & & & 0.142 & & & & 1.000 & & & & 0.367 \\
\hline No & 79 & 33 & 46 & & 29 & 9 & 20 & & 108 & 42 & 66 & \\
\hline Yes & 8 & 1 & 7 & & 5 & 2 & 3 & & 13 & 3 & 10 & \\
\hline Tumor diameter & & & & $<0.001$ & & & & 0.070 & & & & $<0.001$ \\
\hline$<5 \mathrm{~cm}$ & 18 & 14 & 4 & & 6 & 4 & 2 & & 24 & 18 & 6 & \\
\hline$\geq 5 \mathrm{~cm}$ & 69 & 20 & 49 & & 28 & 7 & 21 & & 97 & 27 & 70 & \\
\hline
\end{tabular}

AFP: alpha-fetoprotein; HBVDNA: hepatitis B virus-dexoyribonucleic acid.

TABLE 2: Cox regression analyses of factors predicting disease-free survival and overall survival of HCC.

\begin{tabular}{|c|c|c|c|c|c|c|c|c|c|}
\hline \multirow{2}{*}{ Characteristic } & \multicolumn{3}{|c|}{ Training set } & \multicolumn{3}{|c|}{ Validation set } & \multicolumn{3}{|c|}{ Combined set } \\
\hline & HR & $95 \% \mathrm{CI}$ & $P$ value & HR & $95 \% \mathrm{CI}$ & $P$ value & HR & $95 \% \mathrm{CI}$ & $P$ value \\
\hline \multicolumn{10}{|l|}{ DFS } \\
\hline Gender & 1.174 & $0.506-2.727$ & 0.708 & 1.024 & $0.446-2.355$ & 0.955 & 2.447 & $0.874-6.849$ & 0.088 \\
\hline Age & 1.022 & $0.406-2.576$ & 0.963 & 1.642 & $0.310-8.701$ & 0.560 & 0.521 & $0.193-1.401$ & 0.196 \\
\hline Family history & 1.687 & $0.681-4.181$ & 0.259 & 1.510 & $0.273-8.371$ & 0.637 & 0.733 & $0.305-1.761$ & 0.487 \\
\hline AFP & 1.691 & $0.876-3.266$ & 0.118 & 0.425 & $0.104-1.740$ & 0.234 & 1.632 & $0.865-3.080$ & 0.131 \\
\hline HBVDNA & 2.353 & $1.038-5.337$ & 0.041 & 0.546 & $0.135-2.204$ & 0.395 & 2.010 & $1.019-3.965$ & 0.044 \\
\hline Metastasis & 1.226 & $0.482-3.116$ & 0.668 & 2.370 & $0.502-11.177$ & 0.276 & 1.129 & $0.519-2.456$ & 0.760 \\
\hline Tumor diameter & 1.515 & $0.557-4.117$ & 0.416 & 4.458 & $0.466-42.604$ & 0.194 & 0.956 & $0.421-2.171$ & 0.914 \\
\hline FOXP4-AS1 expression & 2.574 & $1.126-5.885$ & 0.025 & 6.826 & $1.228-37.945$ & 0.028 & 2.548 & 1.249-5.195 & 0.010 \\
\hline \multicolumn{10}{|l|}{ OS } \\
\hline Gender & 1.208 & $0.522-2.796$ & 0.659 & 1.163 & $0.498-2.718$ & 0.727 & 1.384 & $0.617-3.101$ & 0.430 \\
\hline Age & 1.036 & $0.408-2.626$ & 0.941 & 1.689 & $0.327-8.732$ & 0.532 & 0.643 & $0.215-1.919$ & 0.849 \\
\hline Family history & 1.399 & $0.566-3.459$ & 0.468 & 2.248 & $0.406-12.454$ & 0.354 & 1.474 & $0.683-3.184$ & 0.323 \\
\hline AFP & 1.727 & $0.888-3.361$ & 0.107 & 0.312 & $0.077-1.259$ & 0.102 & 1.317 & $0.740-2.344$ & 0.349 \\
\hline HBVDNA & 3.086 & $1.366-7.127$ & 0.008 & 0.384 & $0.091-1.622$ & 0.193 & 1.676 & $0.864-3.250$ & 0.127 \\
\hline Metastasis & 2.003 & $0.772-5.198$ & 0.153 & 2.829 & $0.602-13.285$ & 0.188 & 1.309 & $0.612-2.801$ & 0.487 \\
\hline Tumor diameter & 1.204 & $0.418-3.465$ & 0.730 & 6.600 & $0.633-68.799$ & 0.115 & 1.667 & $0.674-4.120$ & 0.269 \\
\hline FOXP4-AS1 expression & 2.712 & $1.140-6.450$ & 0.024 & 6.505 & $1.165-36.399$ & 0.033 & 3.012 & $1.441-6.297$ & 0.003 \\
\hline
\end{tabular}

AFP: alpha-fetoprotein; HBVDNA: hepatitis B virus-dexoyribonucleic acid. 

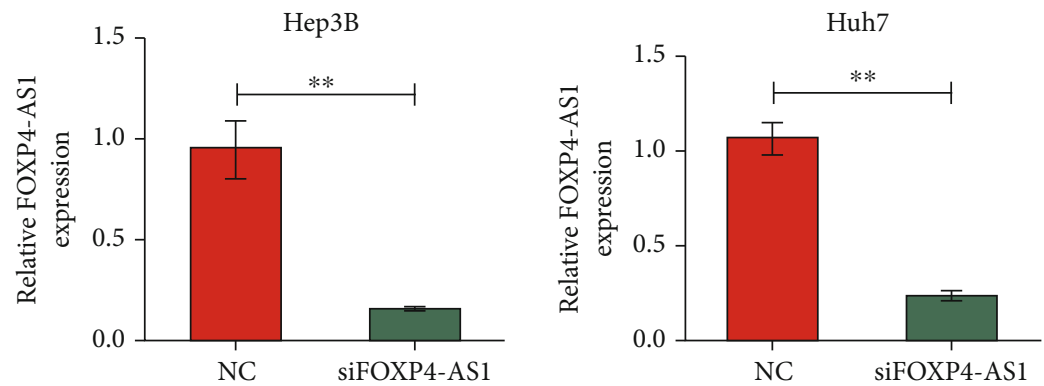

(a)
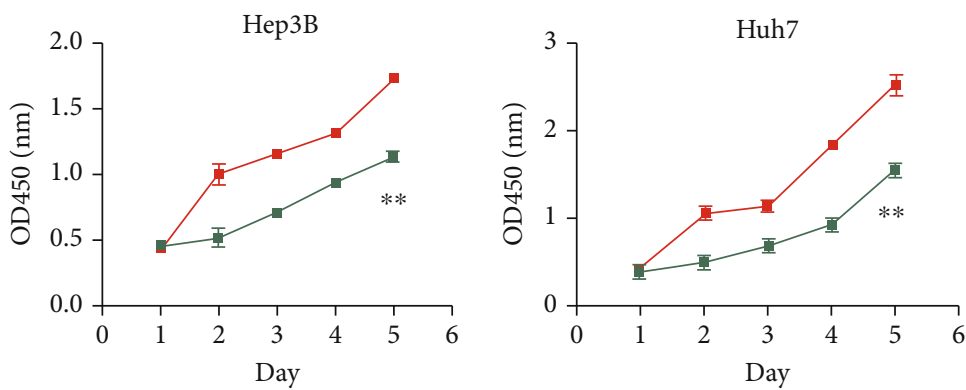

$\rightarrow \mathrm{NC}$

$\rightarrow$ siFOXP4-AS1

(b)
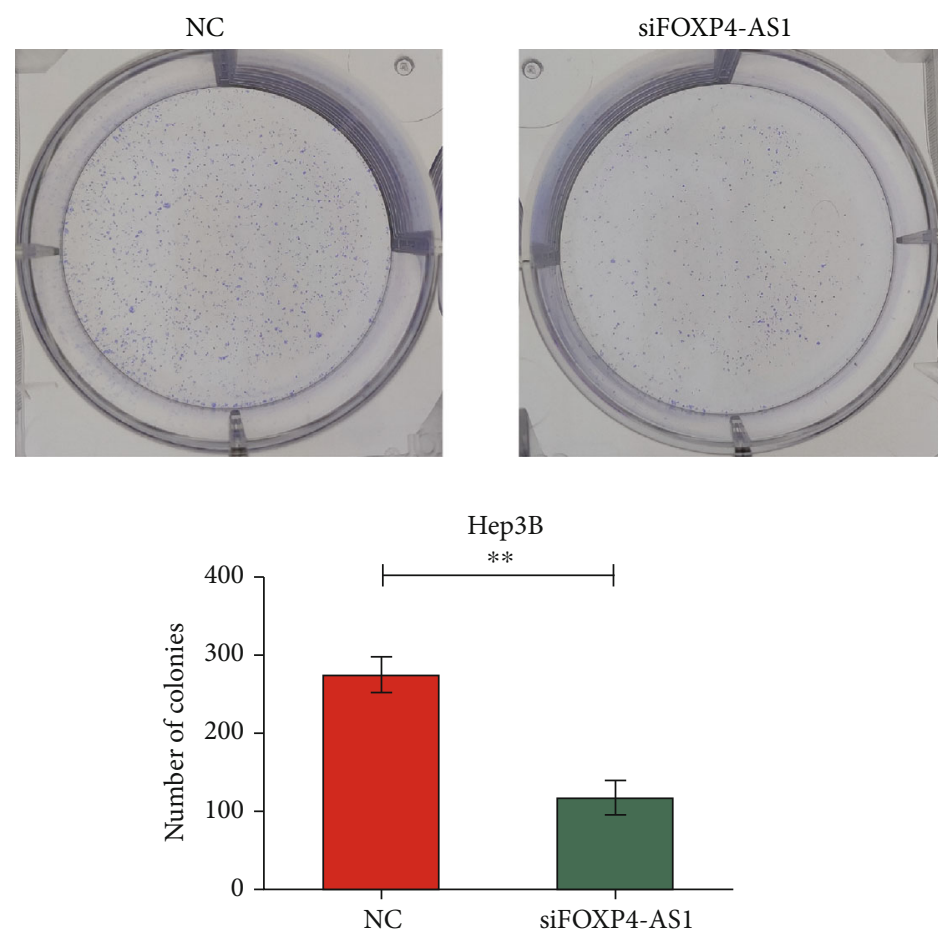

(c)

Figure 2: Continued. 

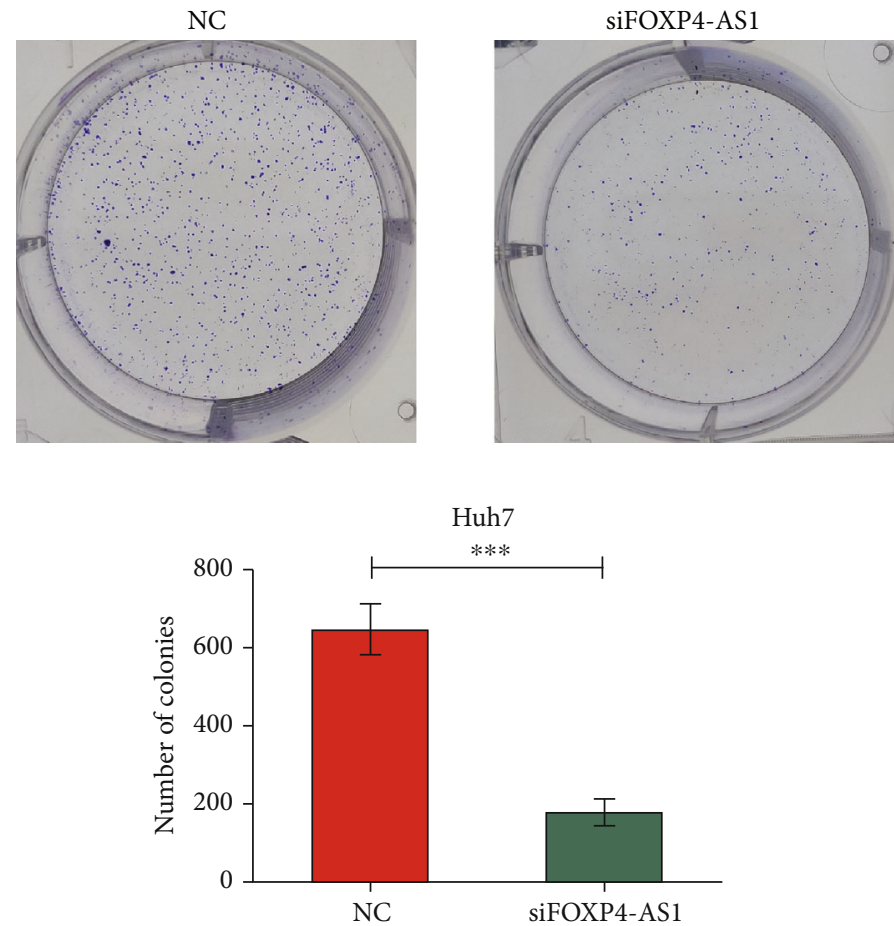

(d)

Figure 2: Effect of FOXP4-AS1 on the proliferation of hepatoma cells. (a) Silencing effect of siRNA on FOXP4-AS1 in Hep3B and Huh7 cells. (b-d) The proliferation of Hep3B and Huh7 cells treated with NC or si-FOXP4-AS1 was detected by CCK8 or colony-forming assay. All data are representative of three independent experiments and presented as mean \pm s.d.

\section{Results}

3.1. Upregulation of FOXP4-AS1 Expression in Patients with HCC. The expression of FOXP4-AS1 was detected in 121 cases of HCC tissues and adjacent normal liver tissues. Meanwhile, the combination set of the training set and validation set was also analysed. We then compared gene expression in HCC tissues and adjacent normal tissues of each patient and divided each group into high- and lowexpression groups (Figures 1(a)-1(c)). For the training set, the expression level of FOXP4-AS1 in HCC tissues was considerably upregulated compared with the adjacent nontumour tissues $(P<0.001$, Figure $1(\mathrm{~d}))$. For the validation dataset, the expression of FOXP4-AS1 was also evidently increased $(P<0.001$, Figure 1(e)). These are consistent with the results of the combined group $(P<0.001$, Figure $1(\mathrm{f}))$.

3.2. Correlation between FOXP4-AS1 and Clinical Parameters. We built the relationship between the FOXP4AS1 expression level and clinical symptoms. In the training group, the FOXP4-AS1 level in HCC samples was associated with age $(P=0.001)$, alpha-fetoprotein (AFP) $(P=0.002)$, and tumour diameter $(P<0.001)$. FOXP4-AS1 level was strikingly related to age, AFP, and tumour diameter ( $P=0.001, P<0.001$, and $P<0.001$, respectively) in the combined set (Table 1). Subsequently, the disease-free survival (DFS) and overall survival (OS) of different groups were compared according to the FOXP4-AS1 level. Kaplan-Meier analysis revealed that patients with higher
FOXP4-AS1 level in the training, verification, and combined sets had shorter DFS $(P<0.001, P=0.011$, and $P<0.001$, respectively; Figures $1(\mathrm{~g})-1(\mathrm{i}))$. Similarly, the patients in the 3 groups with higher FOXP4-AS1 revealed poor OS values $(P=0.006, P=0.023$, and $P<0.001$, respectively; Figures $1(\mathrm{j})-1(\mathrm{l}))$.

Besides, in the multivariable Cox regression analysis, in the training group, FOXP4-AS1 was an independent predictor for DFS (hazard ratio (HR), 2.574; 95\% confidence interval $(\mathrm{CI}), 1.126-5.885 ; P=0.025)$ and $\mathrm{OS}(\mathrm{HR}, 2.712 ; 95 \% \mathrm{CI}$, $1.140-6.450 ; P=0.024)$. In the validation group, FOXP4-AS1 overexpression was an independent predictor for both DFS and OS (HR, 6.826; 95\% CI, 1.228-37.945; DFS, $P=0.028$; $\mathrm{HR}, 6.505$; 95\% CI, 1.165-36.399; OS, $P=0.033)$. In the combined set, the FOXP4-AS1 level was an independent predictor for DFS and OS (HR, 2.548; 95\% CI, 1.249-5.195; DFS, $P=0.010$; HR, 3.012; 95\% CI, 1.441-6.297; OS, $P=0.003$ ) (Table 2). Taken together, a high FOXP4-AS1 expression may be associated with poor prognosis of patients with HCC.

3.3. Downregulation of FOXP4-AS1 Inhibited HCC Cell Proliferation, Migration, and Invasion. The silencing effect of siRNA was validated in the Hep3B and Huh7 cell lines. We defined the nonsilenced group as the NC group (control group) and the silencing group as the siFOXP4-AS1 group. The RT-PCR results revealed that the siRNA designed by us had a significant silencing effect on both cell lines $(P<0.01$, Figure $2(\mathrm{a}))$. Then, the results of CCK- 8 indicated that downregulated FOXP4-AS1 obviously restrained the cell 

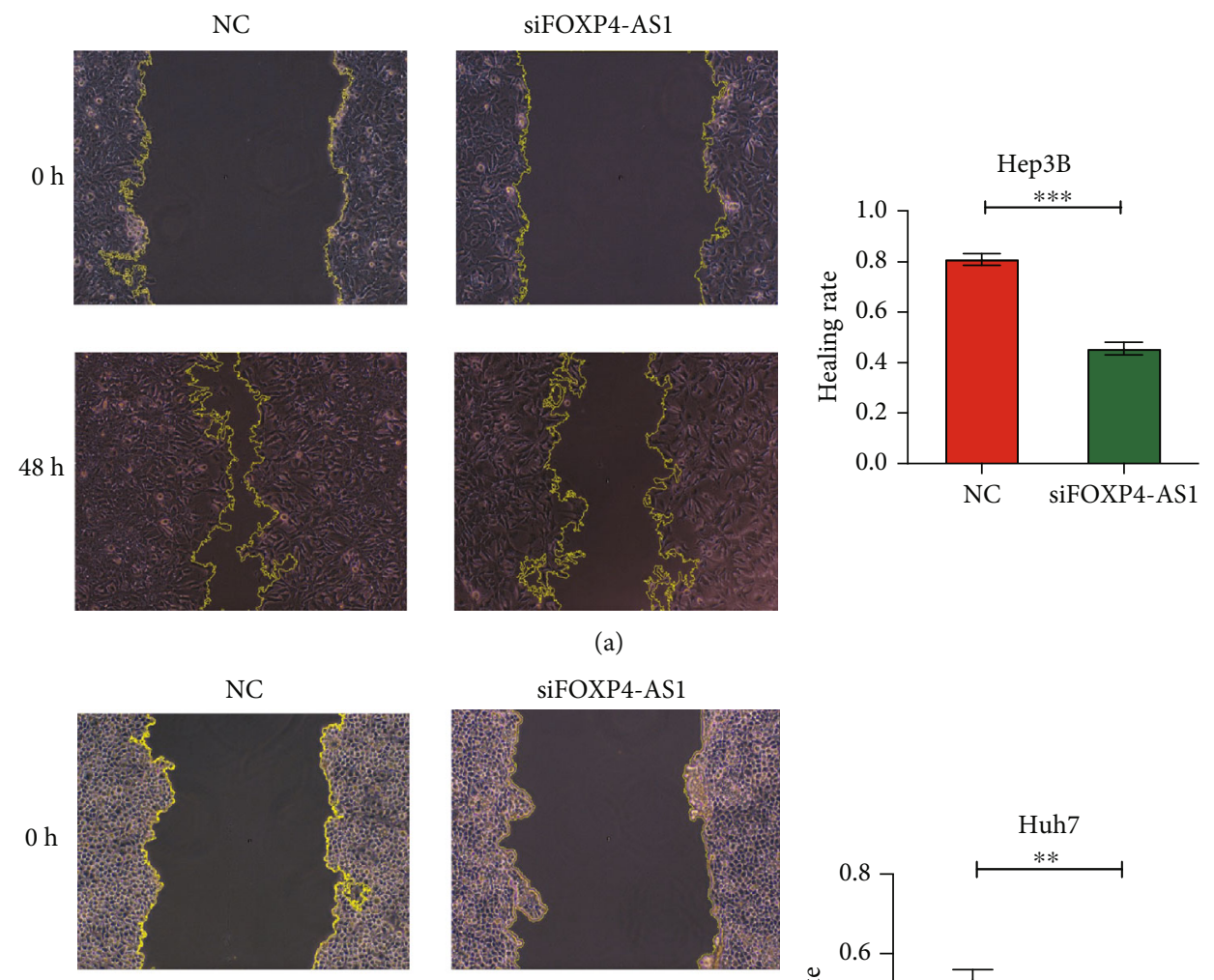

siFOXP4-AS1
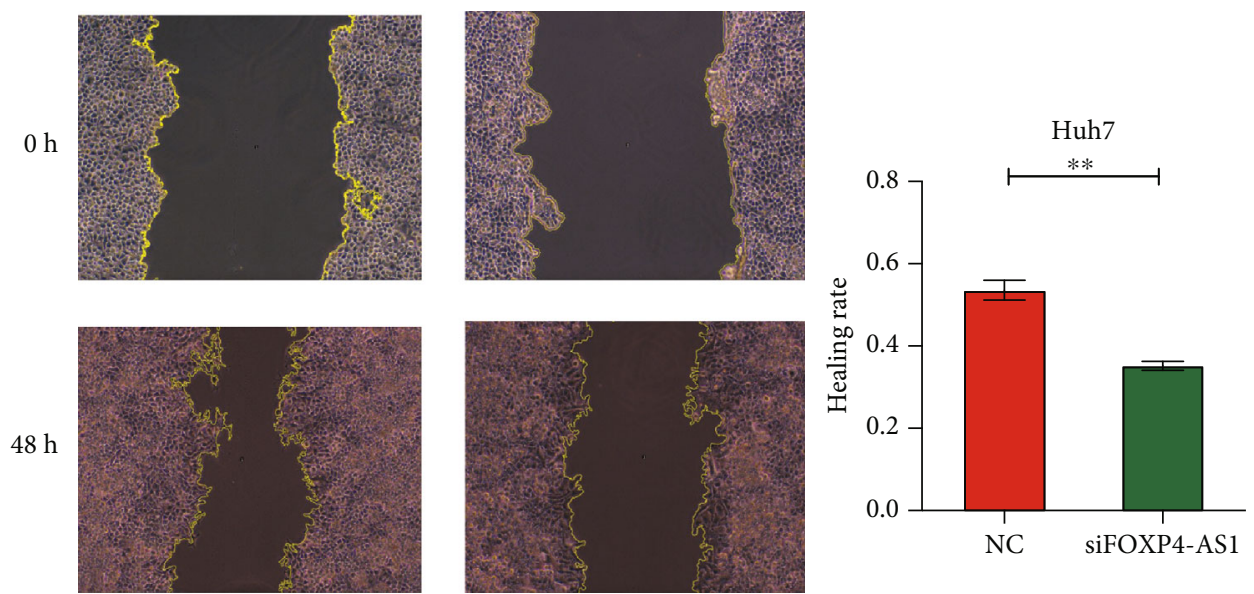

(b)
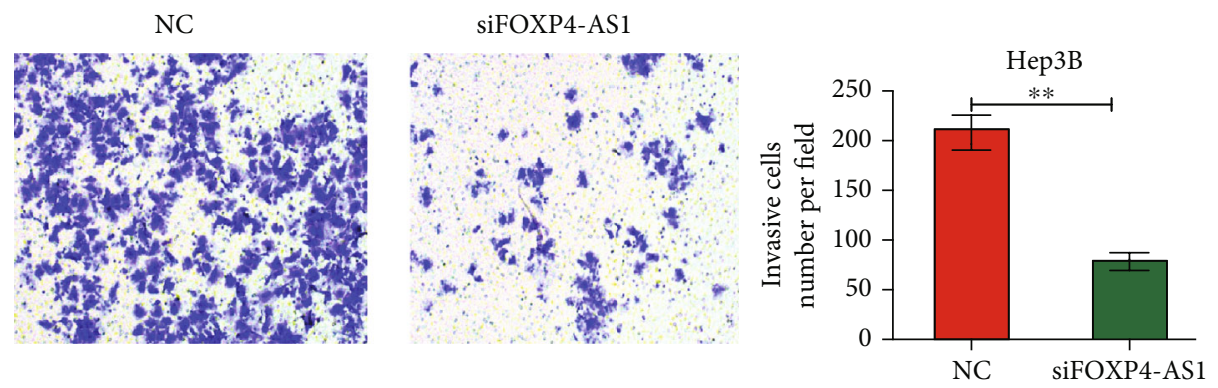

(c)

NC

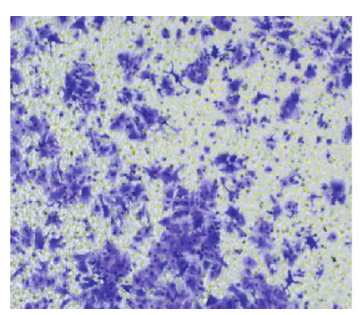

siFOXP4-AS1
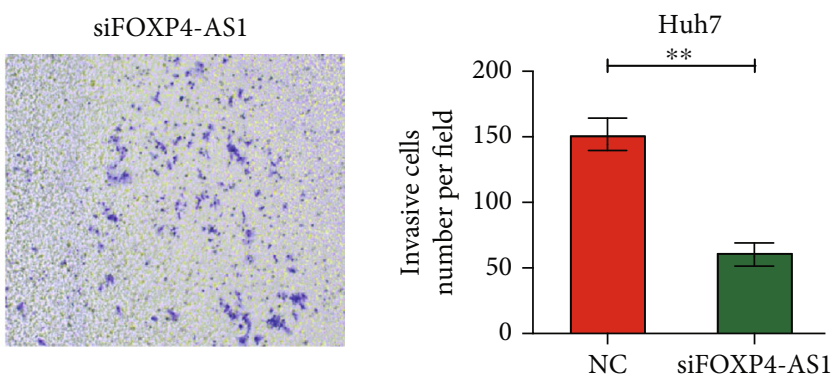

(d)

Figure 3: Continued. 

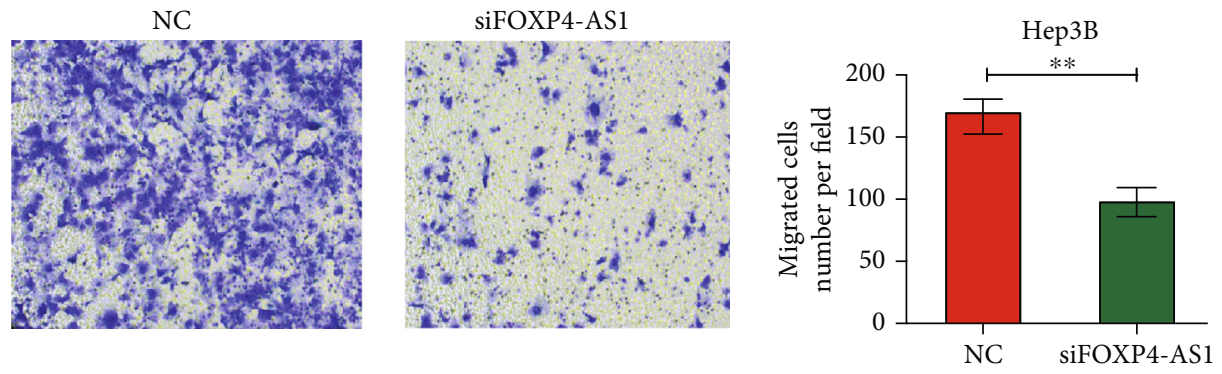

(e)
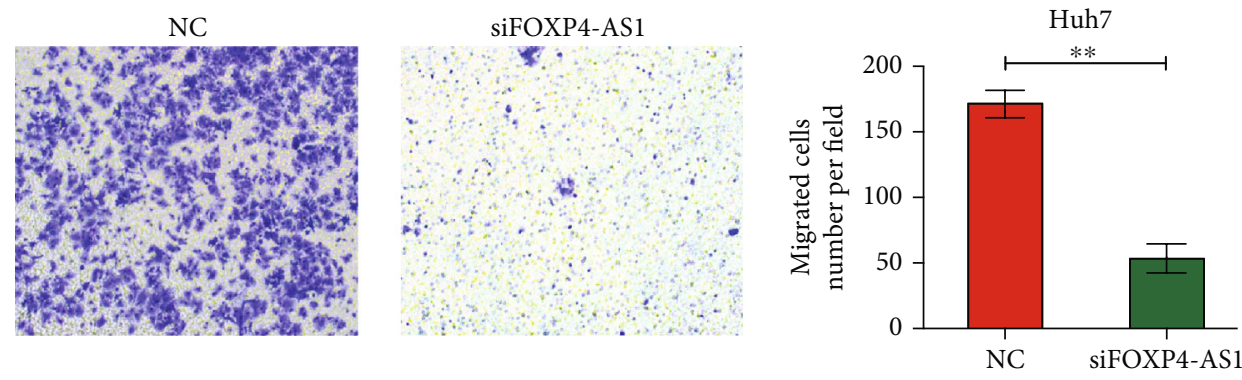

(f)

Figure 3: Effects of FOXP4-AS1 on Hep3B and Huh7 cells migration and invasion in vitro. (a, b) Cell scratch assay was used to detect the effect of FOXP4-AS1 on cell migration. The effects on cell migration and invasion were determined with the cell transwell test. (c) Detection of Hep3B cell invasion. (d) Detection of Huh7 cell invasion. (e) Hep3B cell migration assay. (f) Huh7 cell migration assay. All data are representative of three independent experiments and presented as mean \pm s.d.

proliferation $(P<0.01$, Figure $2(\mathrm{~b}))$. Similarly, colony formation assay indicated that knockdown of FOXP4-AS1 inhibited the colony numbers of Hep3B $(P<0.01$, Figure $2(\mathrm{c}))$ and Huh7 $(P<0.001$, Figure $2(\mathrm{~d}))$ cells.

Moreover, we investigated the effect of FOXP4-AS1 on the migration and invasion of hepatoma cells by scratch test and transwell analysis. The scratch experimental results revealed that the migration abilities of the Hep3B $(P<0.001$, Figure 3(a)) and Huh7 $(P<0.01$, Figure 3(b)) HCC lines decreased evidently after FOXP4-AS1 was knocked down. Transwell assay displayed that the migration and invasion ability of the siFOXP4-AS1 group were remarkably lower than those of the NC group $(P<0.01$, Figures 3(c)-3(f)). These results manifested that the FOXP4-AS1 downregulation inhibited the proliferation, migration, and invasion of the HCC cells.

3.4. Knockdown of FOXP4-AS1 Inhibited Angiogenesis. HUVECs were cultured with different supernatants of transfected cells to validate the effect of FOXP4-AS1 on neovascularisation. It was observed that the angiogenesis abilities of the siFOXP4-AS1 group were obviously weaker than the NC group, and the number of vascular nodes, crossing points, mesh number, vascular branches, and trunk length differed significantly $(P<0.05$, Figure 4$)$.

\section{Discussion}

In the past decade, HCC has become one of the most common malignancies. Currently, the therapy for advanced HCC includes surgery, chemotherapy, and targeted drug therapy. However, the prognosis of patients with HCC remains pessimistic owing to the high recurrence rate and chemotherapy resistance [9]. Therefore, it is urgent to explore the mechanism of the HCC progression and explore new prognostic predictors.

lncRNAs are a class of noncoding RNA transcripts with $>200$ nucleotides [10]. As a pivotal regulator of certain genes and signal pathways related to tumourigenesis, mounting evidence indicates that lncRNAs are associated with numerous cell biological processes, incorporating cell proliferation, autophagy, apoptosis, invasion and metastasis, metabolism, and exosome secretion [11-15]. Emerging research has revealed that the deregulation of $\operatorname{lncRNAs}$ plays a part in the occurrence and development of HCC. Many lncRNAs such as LINC01419 [16], MFI2-AS1 [17], and LINC00160 [18] have been reported to be upregulated in HCC. Consistent with these IncRNAs, FOXP4-AS1 was greatly overexpressed in HCC tissues and cells in this study. Furthermore, lncRNAs are also used to predict the prognosis of multiple tumours. For example, lncRNA MNX1-AS1 and LINC00346 are upregulated in gastric cancer and predict poor prognosis $[19,20]$; overexpression of $\operatorname{lncDQ}$ manifests low OS of patients with HCC [21]. Wu et al. [22] reported in their study that lncRNA FOXP4-AS1 is a prognostic indicator in prostate cancer. Actually, the worth of lncRNAs on evaluating the prognosis of patients with HCC could contribute guidance for postoperative adjuvant therapy. However, the clinical and potential biological functions of FOXP4-AS1in HCC have not been completely reported. Our results revealed that FOXP4-AS1 was highly expressed in HCC and was an independent risk factor for DFS and OS in patients with HCC, indicating that FOXP4-AS1 could be counted as a marker to predict the prognosis of HCC. 
(a)

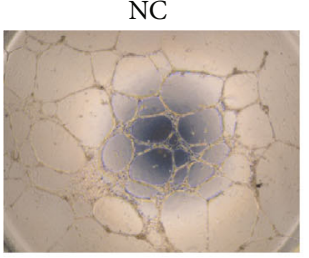

(b)

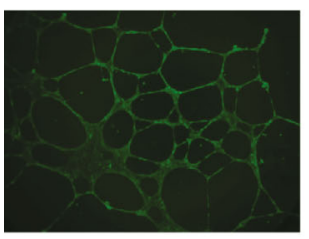

(c)

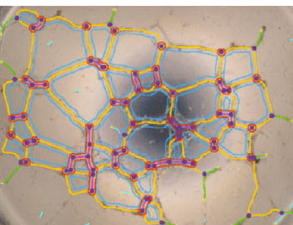

siFOXP4-AS1
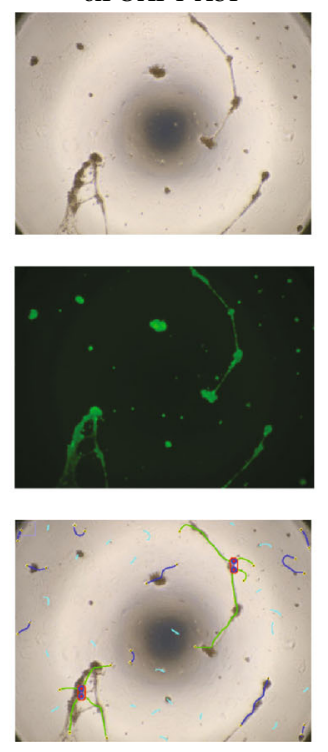

ep3B
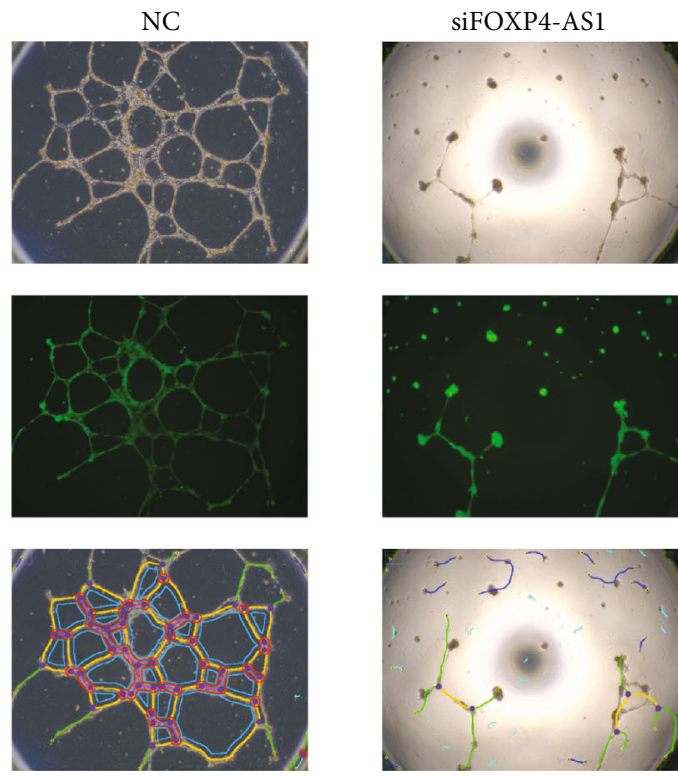

Huh7

FIGURE 4: Detection of FOXP4-AS1 affecting neovascularisation ability. (a) Light microscope image. (b) Fluorescence image. (c) Computer postprocessing image.

Furthermore, the abnormal expression of lncRNAs is closely related to tumour differentiation, proliferation, and metastasis [23]. Accumulating studies indicate that lncRNAs play a vital role in many biological and behavioural processes (cell proliferation, migration, invasion, and angiogenesis) of HCC [24-26]. For example, Zhan et al. [27] found that HOXA11-AS can promote the proliferation and invasion of HCC and induce epithelial-mesenchymal transformation. Besides, Kong et al. [28] confirmed in their research that silencing OTUD6B-AS1 obviously reduced the proliferation and invasion of HCC cells. In contrast, the overexpression of otud6b-as1 produced the contrary results. In addition, the study by Wang et al. provided proofs that angiogenesis in HCC is impeded by the silencing of BZRAP1-AS1. Our experimental results demonstrate that FOXP4-AS1 can significantly increase the cloning and proliferation abilities of HCC cells. The siFOXP4-AS1 group showed significant decreases in invasion, migration, and angiogenesis compared with the NC group. Therefore, in the future, this in-depth study of FOXP4-AS1 may be used to improve the high recurrence rate and high metastasis rate of patients with HCC. The cell function experiment fully confirmed the biological effect of FOXP4-AS1 on the growth and metastasis of liver cancer cells. Future studies will continue to evaluate the specific mechanism and pathway of FOXP4-AS1. As previously mentioned, FOXP4-AS1 is a FOXP4 antisense RNA; past studies have also pointed out that it will induce the expression of FOXP4. For example, it has been reported that lncRNA FOXP4-AS1 promotes the growth of prostate cancer by sequestering miR-3184-5p to upregulate FOXP4 [22]. In addition, FOXP4-AS1 positively regulated FOXP4 by interacting with insulin-like growth factor 2 mRNA-binding protein 2 (IGF2BP2) to stabilize FOXP4 mRNA in esophageal squamous cell carcinoma [29]. Interestingly, Zhang and
Zhang discovered that FOXP4 was highly expressed in HCC and could promote (epithelial-mesenchymal transition) EMT of HCC cell line by regulating slug [30]. However, the role and molecular mechanism of FOXP4-AS1 and FOXP4 in hepatocellular carcinoma are still unclear. Our study confirmed that FOXP4-AS1 is a prognostic factor and affects the biological function of HCC. Further studies are needed to verify the specific mechanism of FOXP4-AS1 regulation and the correlation between FOXP4-AS1 and FOXP4 in HCC. In addition, our results need to be further verified in vivo. We will continue to explore in future experiments.

\section{Conclusion}

In summary, our study showed that FOXP4-AS1 overexpression in HCC is closely related to the prognosis of HCC. Moreover, the downregulation of FOXP4-AS1 inhibited the proliferation, invasion, and migration capacities of HCC, suggesting that FOXP4-AS1 plays a role as an oncogene in the occurrence and development of HCC cells, which provides a novel orientation for finding new therapeutic targets and potential prognostic biomarkers of HCC.

\section{Data Availability}

The data used to support the findings of this study is available from the corresponding author upon request.

\section{Conflicts of Interest}

The authors declare that they have no competing interests. 


\section{Authors' Contributions}

Pingping Guo, Hang Li, and Junjie Liu designed the study. Duo Wang participated in the experiment of the research. Jingchen Liang contributed to the writing of the manuscript. Guanhua Qiu collected and analyzed the data. Xiaoqi Zhu analyzed the data and interpreted the results. All authors have read and approved the manuscript. Jingchen Liang, Duo Wang, and Guanhua Qiu contributed equally to this work.

\section{Acknowledgments}

This study was financially supported by the Guangxi Natural Science Foundation(2017GXNSFBA198234 and Grant No. AB18221103).

\section{References}

[1] E. Tsochatzis, T. Meyer, and A. Burroughs, "Hepatocellular carcinoma," Lancet, vol. 3, p. 55, 1995.

[2] H. Z. Sun, Y. L. Song, and X. Y. Wang, "Effects of different anesthetic methods on cellular immune and neuroendocrine functions in patients with hepatocellular carcinoma before and after surgery," Journal of Clinical Laboratory Analysis., vol. 30, no. 6, pp. 1175-1182, 2016.

[3] L. Lorenzi, F. A. Cobos, A. Decock et al., "Long non-coding RNA expression profiling in cancer: challenges and opportunities," Genes Chromosomes \& Cancer, vol. 58, no. 4, pp. 191199, 2019.

[4] T. Derrien, R. Johnson, G. Bussotti et al., "The GENCODE v7 catalog of human long noncoding RNAs: analysis of their gene structure, evolution, and expression," Genome Research, vol. 22, no. 9, pp. 1775-1789, 2012.

[5] K. C. Wang and H. Y. Chang, "Molecular mechanisms of long noncoding RNAs," Molecular Cell, vol. 43, no. 6, pp. 904-914, 2011.

[6] W. Sun, Y. Yang, C. Xu, and J. Guo, "Regulatory mechanisms of long noncoding RNAs on gene expression in cancers," Cancer Genetics, vol. 216-217, pp. 105-110, 2017.

[7] J. Zhao, T. Yang, and L. Li, "LncRNA FOXP4-AS1 is involved in cervical cancer progression via regulating miR-1365p/CBX4 axis," OncoTargets and Therapy, vol. Volume 13, pp. 2347-2355, 2020.

[8] J. Li, Y. Lian, C. Yan et al., "Long non-coding RNA FOXP4AS1 is an unfavourable prognostic factor and regulates proliferation and apoptosis in colorectal cancer," Cell Proliferation, vol. 50, no. 1, 2017.

[9] J. Hindson and T. A. C. E. Combined, "And sorafenib for HCC treatment," Nature Reviews Gastroenterology \& Hepatology, vol. 17, no. 2, 2020 .

[10] H. Ling, K. Vincent, M. Pichler, R. Fodde, G. A. Calin, and D. N. A. Junk, "Junk DNA and the long non-coding RNA twist in cancer genetics," Oncogene, vol. 34, no. 39, pp. 5003-5011, 2015.

[11] W. Huang, F. Huang, Z. Lei, and H. Luo, "LncRNA SNHG11 promotes proliferation, migration, apoptosis, and autophagy by regulating hsa-miR-184/AGO2 in HCC," Oncotargets \& Therapy, vol. Volume 13, pp. 413-421, 2020.

[12] T. Guo, C. Gong, P. Wu, S. F. Battaglia-Hsu, and Z. Li, "LINC00662 promotes hepatocellular carcinoma progression via altering genomic methylation profiles," Cell Death \& Differentiation, vol. 27, no. 7, pp. 2191-2205, 2020.

[13] Y. F. Guan, Q. L. Huang, Y. L. Ai et al., "Nur77-activated lncRNA WFDC21P attenuates hepatocarcinogenesis via modulating glycolysis," Oncogene, vol. 39, no. 11, pp. 2408-2423, 2020.

[14] L. Yang, X. Peng, Y. Li et al., "Long non-coding RNA HOTAIR promotes exosome secretion by regulating RAB35 and SNAP23 in hepatocellular carcinoma," Molecular Cancer, vol. 18 , no. 1 , p. $78,2019$.

[15] T. Luo, M. Chen, Y. Zhao et al., "Macrophage-associated lncRNA ELMO1-AS1: a novel therapeutic target and prognostic biomarker for hepatocellular carcinoma," OncoTargets and Therapy, vol. Volume 12, pp. 6203-6216, 2019.

[16] H. Dang, L. Chen, P. Tang et al., "LINC01419 promotes cell proliferation and metastasis in hepatocellular carcinoma by enhancing NDRG1 promoter activity," vol. 43, no. 5, pp. 931-947, 2020.

[17] Y. Wei, Z. Wang, Y. Zong, D. Deng, and J. Lu, "lncRNA MFI2AS1 promotes HCC progression and metastasis by acting as a competing endogenous RNA of miR-134 to upregulate FOXM1 expression," Biomedicine \& Pharmacotherapy, vol. $125,2020$.

[18] Y. Peng, D. Tang, M. Zhao, H. Kajiyama, F. Kikkawa, and Y. Kondo, "Long non-coding RNA: a recently accentuated molecule in chemoresistance in cancer," Cancer and Metastasis Reviews., vol. 39, no. 3, pp. 825-835, 2020.

[19] W. Zhang, L. Huang, X. Lu, K. Wang, and Z. Liu, "Upregulated expression of MNX1-AS1 long noncoding RNA predicts poor prognosis in gastric cancer," Bosnian Journal of Basic Medical Sciences, vol. 19, 2019.

[20] T. P. Xu, P. Ma, W. Y. Wang et al., "KLF5 and MYC modulated LINC00346 contributes to gastric cancer progression through acting as a competing endogeous RNA and indicates poor outcome," Cell Death \& Differentiation, vol. 26, no. 11, pp. 21792193, 2019.

[21] B. Zeng, Z. Lin, H. Ye et al., "Upregulation of lncDQ is associated with poor prognosis and promotes tumor progression via epigenetic regulation of the EMT pathway in HCC," Cellular Physiology \& Biochemistry, vol. 46, no. 3, pp. 1122-1133, 2018.

[22] X. Wu, Y. Xiao, Y. Zhou, Z. Zhou, and W. Yan, "lncRNA FOXP4-AS1 is activated by PAX5 and promotes the growth of prostate cancer by sequestering miR-3184-5p to upregulate FOXP4," Cell Death \& Disease, vol. 10, no. 7, 2019.

[23] S. Geisler and J. Coller, "RNA in unexpected places: long noncoding RNA functions in diverse cellular contexts," Nature Reviews Molecular Cell Biology, vol. 14, no. 11, pp. 699-712, 2013.

[24] J. Lin, S. Cao, W. Yu et al., "Long non-coding RNA UBE2CP3 enhances HCC cell secretion of VEGFA and promotes angiogenesis by activating ERK1/2/HIF-1 $\alpha$ /VEGFA signalling in hepatocellular carcinoma," Journal of Experimental \& Clinical Cancer Research, vol. 37, no. 1, 2018.

[25] L. Deng, S.-B. Yang, F.-F. Xu, and J.-H. Zhang, "Long noncoding RNA CCAT1 promotes hepatocellular carcinoma progression by functioning as let-7 sponge," Journal of Experimental \& Clinical Cancer Research, vol. 34, no. 1, 2015.

[26] J. J. Quinn and H. Y. Chang, "Unique features of long noncoding RNA biogenesis and function," Nature Reviews Genetics, vol. 17, no. 1, pp. 47-62, 2015. 
[27] M. Zhan, K. He, J. Xiao et al., "LncRNA HOXA11-AS promotes hepatocellular carcinoma progression by repressing miR-214-3p," Journal of Cellular \& Molecular Medicine, vol. 22, no. 8, pp. 3758-3767, 2018.

[28] S. Kong, H. Xue, Y. Li et al., "The long noncoding RNA OTUD6B-AS1 enhances cell proliferation and the invasion of hepatocellular carcinoma cells through modulating GSKIP/Wnt/ $\beta$-catenin signalling via the sequestration of miR-664b-3p," Experimental Cell Research, vol. 395, no. 1, 2020.

[29] Y. Li, T. Li, Y. Yang, W. Kang, S. Dong, and S. Cheng, "YY1induced upregulation of FOXP4-AS1 and FOXP4 promote the proliferation of esophageal squamous cell carcinoma cells," Cell biology international, vol. 44, no. 7, pp. 1447-1457, 2020.

[30] G. Zhang and G. Zhang, "Upregulation of FoxP4 in HCC Promotes Migration and Invasion through Regulation of EMT," Oncology letters, vol. 17, no. 4, pp. 3944-3951, 2019. 